\title{
Visual Snow Syndrome and Its Relationship to Tinnitus
}

Matthew Renze

\begin{abstract}
Visual snow is a symptom described as the continuous perception of tiny flickering dots in the entire field of vision, similar to static of an analog television. Visual snow syndrome is a cluster of symptoms found highly prevalent in patients that present with visual snow. While most of these symptoms appear to be visual in nature, approximately $63 \%$ of patients studied also report continuous bilateral tinnitus. The high correlation of visual-snow-syndrome patients presenting with tinnitus suggests that they may share a common underlying pathophysiology.
\end{abstract}

Keywords: visual snow, visual snow syndrome, tinnitus.

Data Science Consultant, Renze Consulting, United States, E-mail: matthew@matthewrenze.com 
Visual Snow is a symptom described as the continuous perception of tiny flickering dots in the entire field of vision, similar to static of an analog television ${ }^{1}$. Visual Snow Syndrome is a cluster of symptoms found highly prevalent in patients that present with visual snow ${ }^{2}$. While most of these symptoms appear to be visual in nature, $62-64 \%$ of patients studied also report continuous bilateral tinnitus ${ }^{1-3}$.

In 2014, Schankin et al. ${ }^{1}$ attempted to characterize the disorder using a chart review of 22 patients with visual snow, an internet survey of 275 self-assessed visualsnow subjects, and a phone interview with an additional 142 patients. This study demonstrated that visual snow syndrome was a unique disorder distinct from migraine and typical migraine with aura.

This study by Schankin et al. was the first attempt to systematically identify additional symptoms commonly found with visual snow. The cluster of visual symptoms includes palinopsia (seeing afterimages), entopic phenomena (excessive floaters, blue-field entoptic phenomena, spontaneous photopsia), photophobia (sensitivity to bright light), and nyctalopia (impaired night vision). However, in addition to visual symptoms, $62 \%$ of the 78 phone-interview patients with confirmed visual snow also reported continuous bilateral non-pulsatile tinnitus.

An additional study by Schankin et al. ${ }^{2}$, provided the first empirical evidence that visual snow syndrome was an objectively measurable disorder. Using functional brainimaging techniques (positron emission tomography), this study detected areas of increased metabolic activity in the right lingual gyrus and the anterior lobe of the left cerebellum of 17 patients with visual snow, compared to healthy control subjects. In addition, this second study expanded the previous telephone survey to 120 patients with confirmed visual snow. Of these patients, $64 \%$ reported continuous bilateral tinnitus ${ }^{2}$.

In 2016 Lauschke et al. ${ }^{3}$ proposed that visual snow syndrome may be a thalamocortical dysrhythmia of the visual pathway; specifically, a thalamocortical dysrhythmia secondary to dysfunctional neuronal excitability and impaired habituation response. They proposed that an imbalance between the konio- and parvo/magnocellular pathway underlies the thalamocortical dysrhythmia. The study demonstrated that color filters, primarily in the blueyellow spectrum, subjectively reduced symptoms in $92 \%$ of 12 patients who participated in a colorimetry test. This study suggests that visual snow syndrome may be related to other sensory processing disorders, like tinnitus, via a common pathophysiological mechanism. A connection that has been previously hypothesized between tinnitus and TCD as well ${ }^{4}$. Coincidentally, of the 32 visual snow syndrome patients involved in this study, $63 \%$ reported tinnitus ${ }^{3}$.

Additional hypotheses have also been suggested for how visual snow syndrome and tinnitus may be related. The predictive-coding model, which is a Bayesian-prediction model of sensory processing, suggests a common underlying mechanism between tinnitus and other positive perceptual disorders ${ }^{5}$. In addition, Zambrowski et al. suggest that visual snow syndrome may result from "cerebral hypersensitivity" to physiological phenomena ${ }^{6}$. In essence, visual snow syndrome could be to the visual system what tinnitus is to the auditory system 1 .

There is currently no known treatment for visual snow syndrome ${ }^{1,3}$. In addition, because visual snow syndrome is largely unknown in the medical community, patients are often misdiagnosed as having migraine with aura, hallucinogen persisting perception disorder (HPPD), psychogenic disorders or malingering ${ }^{2,3}$. Additional visual snow syndrome research studies are currently underway in London and Munich ${ }^{7}$. However, funding for this type of research is currently very limited and is largely being self-funded by individuals with self-assessed visual snow syndrome ${ }^{7}$.

While it is currently unknown how visual snow syndrome is related to tinnitus, the high correlation of visual-snow-syndrome patients presenting with tinnitus suggests that they may share a common underlying Pathophysiological mechanism ${ }^{1-3}$. As a result, additional research into visual snow syndrome may provide new insight into the cause of tinnitus and may, hopefully, lead to rational treatment protocols for both visual snow syndrome and tinnitus.

For more information on the relationship between tinnitus and visual snow syndrome, please see the following video on the American Tinnitus Association website: https://www.ata.org/news/news/video-visual-snowsyndrome-relationship-tinnitus

This topic will be discussed in more depth at the International Conference on the Management of the Tinnitus and Hyperacusis Patient, June 15-16, 2017: https://medicine.uiowa.edu/oto/education/conferencesand-events/international-conference-managementtinnitus-and-hyperacusis

\section{REFERENCES}

1. Schankin CJ, Maniyar FH, Digre KB, Goadsby PJ. 'Visual snow'- a disorder distinct from persistent migraine aura. Brain. 2014;137(5):1419-28.

2. Schankin CJ, Maniyar FH, Sprenger T, Chou DE, Eller M, Goadsby PJ. The Relation Between Migraine, Typical Migraine Aura and "Visual Snow". Headache. 2014;54(6):957-66.

3. Lauschke J, Plant G, Fraser C. Visual snow: A thalamocortica dysrhythmia of the visual pathway? J Clin Neurosci. 2016;28:123-7.

4. De Ridder D, Vanneste S, Langguth B, Llinas R. Thalamocortical Dysrhythmia: A Theoretical Update in Tinnitus. Front Neurol. 2015;6:124.

5. Sedley W, Friston K, Gander P, Kumar S, Griffiths T. An Integrative Tinnitus Model Based on Sensory Precision. Trends in Neurosciences. 2016;39(12):799-812.

6. Zambrowski O, Ingster-Moati I, Vignal-Clermont C, Robert M. Le phenomene de neige visuelle. Journal Français d'Ophtalmologie. 2014;37(9):722-7.

7. Ambrose J. Eye on Vision Foundation [Internet]. Eyeonvision.org 2017 [cited 2017 Mar 14]. Available from: http://www.eyeonvision.org/ 